\title{
INFLUENCIA DE LAS PROGRAMACIONES DE RIEGO EN PARCELA SOBRE EL FUNCIONAMIENTO DE UNA RED COLECTIVA DE RIEGO
}

\author{
Lima, F.A. ${ }^{1}$, Córcoles, J.I', Romero, A.M ${ }^{3}$, Tarjuelo, J.M ${ }^{4}$
}

1 Doctorando, Universidad Castilla-La Mancha (UCLM), Centro Regional de Estudios del Agua, aldiel_metal@hotmail.com

2 Dr. Ingeniero Agrónomo, Profesor Ayudante Doctor ElIAB, Departamento de Mecánica Aplicada e Ingeniera de Proyectos, UCLM, juanignacio.corcoles@uclm.es

${ }^{3}$ Dr. Ingeniero Agrónomo, Profesor Asociado; ElIAB, Departamento de Mecánica Aplicada e Ingeniera de Proyectos, UCLM, angel.mromero@uclm.es

${ }^{4}$ Dr. Ingeniero Agrónomo. Catedrático de UCLM. Centro Regional de Estudios del Agua. Albacete. jose.tarjuelo@uclm.es

\section{Resumen}

En zonas regables con redes de distribución de agua, es necesario el desarrollo de herramientas de ayuda para mejorar la eficiencia en el uso del agua y la energía, sin comprometer el rendimiento y productividad de los cultivos. En este trabajo se realiza un análisis del comportamiento de una red de distribución de riego, con distintas estrategias de la programación del riego en parcela, aplicado a un día de máximas necesidades hídricas de los cultivos. La metodología propuesta ha sido aplicada en la zona regable de Tarazona de La Mancha (Albacete, España). Para ello, se establece una programación de riego en diversas parcelas de la zona regable, a partir del año meteorológico típico (TMY). Para evaluar el comportamiento de la red, se han determinado los costes energéticos y consumo de energía en función de los distintos escenarios de apertura de tomas en parcela planteados. Para simular el comportamiento hidráulico de la red, se ha utilizado EPANET ${ }$, en combinación con MATLABß.

\section{Abstract}

In irrigated areas with pressurized irrigation networks, it is necessary to develop tools with the aim of improving efficient use of water and energy resources, maintaining crop yield and productivity. In this paper, the performance of the irrigation network is analyzed, considering several scenarios of on-farm irrigation scheduling for a day with the highest crop water requirements (July). The proposed methodology has been applied to the irrigation area of Tarazona de La Mancha (Albacete). For this aim, an irrigation scheduling was used for each plot of the irrigated area, based on the typical meteorological year (TMY) methodology. To analyze irrigation network performance, energy consumed and energy costs were computed at each scenario of opening of hydrants. To compute hydraulic variables, EPANET® toolkit was used in combination with MATLAB®.

\section{1) Introducción. Objetivo del trabajo.}

En regiones semiáridas como Castilla-La Mancha, donde el régimen pluviométrico es irregular en su distribución temporal y espacial, el regadío llega a ser una actividad necesaria para mantener la rentabilidad de muchas explotaciones. Sin embargo, un manejo incorrecto de los sistemas de riego conlleva repercusiones negativas, tanto económicas como medioambientales. 
Este aspecto adquiere gran relevancia en el caso de redes colectivas de riego, donde es imprescindible mejorar la eficiencia del uso del agua y de la energía como actividad encaminada a garantizar la sostenibilidad del regadío. Esto puede conseguirse implementando estrategias de mejora en la gestión de los recursos hídricos y energéticos en las zonas regables.

Para la gestión de este tipo de zonas, se plantean distintas alternativas de manejo, tales como el riego por turnos o a la demanda. Numerosas herramientas se han desarrollado para la mejora en el manejo de redes colectivas de riego, todas ellas centradas en la reducción del consumo energético de este tipo de instalaciones, entre las que destacan las herramientas para la sectorización de redes (Carrillo-Cobo et al., 2011; Fernández-García et al., 2014) y la determinación de puntos críticos de la red (González Perea et al. 2014).

En el manejo de redes colectivas, los propios agricultores planifican la programación del riego en parcela de forma individual para cada uno de sus cultivos. Por ello, la mejora de la gestión de las redes colectivas de riego, requiere, en la medida de lo posible, la participación de los técnicos responsables de la misma en la programación del riego en parcela, para tratar de optimizar el funcionamiento de la red. Resulta pues imprescindible disponer de un modelo hidráulico de la red y del conjunto de herramientas necesarias para armonizar el manejo del riego en parcela con el funcionamiento de la propia red, que conduzca al mínimo coste total del agua aplicada con el riego.

El objetivo del trabajo es desarrollar una herramienta de ayuda a toma de decisiones, para, partiendo del modelo hidráulico calibrado de una red, analizar su comportamiento utilizando distintas estrategias de programación del riego en parcela.

\section{2) Material y métodos}

\subsection{Caso de estudio}

La metodología propuesta ha sido aplicada en una red de riego a la demanda, perteneciente a la zona regable de Tarazona de La Mancha (Albacete, España) (Fig. 1). La red abastece al sector de estudio, con una superficie regable de 550 ha, donde predomina el sistema de riego por aspersión en cultivos herbáceos y hortícolas. Entre los cultivos más representativos de la zona, con un $50 \%$ de la superficie regable, destacan la cebolla y el maíz. Se trata de una zona que se abastece de recursos hídricos subterráneos, con tres sondeos y un embalse de regulación. La estación de re-bombeo (que conecta el embalse con la red) está compuesta por 10 bombas iguales conectadas en paralelo (140 CV para cada bomba) donde una de ellas está dotada con un variador de velocidad. 


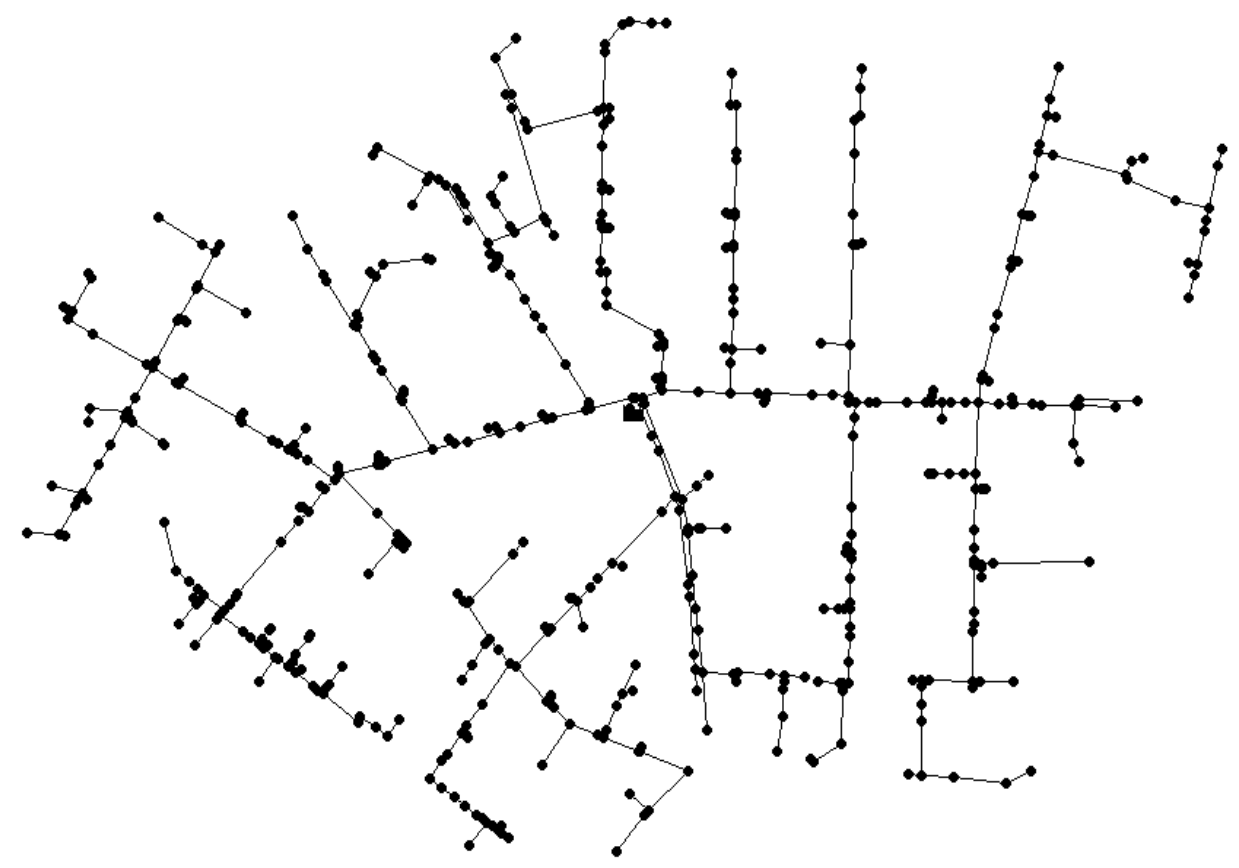

Figura 1. Red de riego de Tarazona de La- Mancha

\subsection{Metodología propuesta}

Para realizar un plan de riegos anual de los cultivos de la zona, se ha partido de la distribución correspondiente a la campaña 2015, con los datos climáticos de un año meteorológico típico (TMY) (Domínguez, et al., 2013) constituido a partir de los datos climáticos de la serie 2000-2014 de una estación de la red del Servicio de información Agroclimática para el Regadío (SIAR) próxima a la zona (MAGRAMA, 2015).

En la Tabla 1 se recogen los datos climáticos del TMY utilizados para establecer la programación de riegos.

Tabla 1. Característica del Año Típico Meteorológico (TMY)

\begin{tabular}{|c|c|c|c|}
\hline Mes & Año & $\begin{array}{c}\text { Precipitación } \\
\text { acumulada }(\mathrm{mm})\end{array}$ & $\begin{array}{c}\text { Evapotranspiración de referencia } \\
\text { (ETo) acumulada }(\mathrm{mm})\end{array}$ \\
\hline Enero & 2002 & 24,8 & 33,7 \\
\hline Febrero & 2012 & 3,2 & 60,9 \\
\hline Marzo & 2005 & 9,0 & 94,1 \\
\hline Abril & 2005 & 30,9 & 132,6 \\
\hline Mayo & 2010 & 39,4 & 132,3 \\
\hline Junio & 2011 & 13,2 & 184,9 \\
\hline Julio & 2009 & 1,4 & 238,4 \\
\hline Agosto & 2009 & 0,0 & 198,1 \\
\hline Septiembre & 2013 & 3,3 & 122,5 \\
\hline Octubre & 2013 & 34,9 & 78,3 \\
\hline Noviembre & 2006 & 39,4 & 65,1 \\
\hline Diciembre & 2011 & 6,0 & 32,4 \\
\hline
\end{tabular}

Una vez conocida la planificación de riegos, se tomó como referencia el día 17 de julio (un día en el período de máximas necesidades hídricas de los cultivos) para realizar las simulaciones de caudales circulantes por la red. Para simplificar, se tuvieron en cuenta sólo 
las parcelas de maíz y cebolla y se consideró un único sector de riego por parcela, estableciendo entonces los escenarios de apertura y cierre de hidrantes. Los volúmenes a aplicar y los tiempos de apertura para cada hidrante durante el día indicado se calcularon a partir de la planificación de riegos establecida, realizando un balance de agua diario en el suelo usando el módulo destinado para este fin en el programa MOPECO (Modelo de Optimización Económica del Riego) (Domínguez, et al., 2012ab) que aplica la metodología FAO (Allen et al., 1998). Los datos incluidos al programa se recogen en la Tabla 2.

Tabla 2. Parámetros utilizados para realizar el balance de agua en el suelo en cebolla y maíz.

\begin{tabular}{|c|c|c|c|c|c|}
\hline \multicolumn{6}{|c|}{ Datos de cultivo } \\
\hline & & \multicolumn{2}{|c|}{ Cebolla } & \multicolumn{2}{|r|}{ Maíz } \\
\hline & & Duración (días) & $\begin{array}{c}\text { Coeficientes } \\
\text { de cultivo } \\
\text { Kc }\end{array}$ & $\begin{array}{c}\text { Duración } \\
\text { (días) }\end{array}$ & $\begin{array}{l}\text { Coeficientes } \\
\text { de cultivo Kc }\end{array}$ \\
\hline \multirow{4}{*}{$\begin{array}{c}\text { Etapa } \\
\text { fenológica }\end{array}$} & Establecimiento & 60 & $0,65-0,65$ & 41 & $0,30-0,30$ \\
\hline & Desarrollo vegetativo & 31 & $0,65-1,20$ & 39 & $0,30-1,10$ \\
\hline & Etapa media & 47 & $1,20-1,20$ & 38 & $1,10-1,10$ \\
\hline & Maduración & 19 & $1,20-0,75$ & 27 & $1,10-0,55$ \\
\hline \multicolumn{2}{|r|}{ Fecha de siembra } & \multicolumn{2}{|c|}{$23 / 03$} & \multicolumn{2}{|c|}{$28 / 04$} \\
\hline \multicolumn{2}{|c|}{ Profundidad Radicular máxima $(\mathrm{cm})$} & \multicolumn{2}{|c|}{40} & \multicolumn{2}{|c|}{40} \\
\hline \multicolumn{2}{|c|}{ Objetivo productivo } & \multicolumn{2}{|c|}{$100 \%$} & \multicolumn{2}{|c|}{$100 \%$} \\
\hline \multicolumn{6}{|c|}{ Datos del suelo } \\
\hline & & \multicolumn{2}{|c|}{ Cebolla } & \multicolumn{2}{|r|}{ Maíz } \\
\hline Profun & didad máxima del suelo $(\mathrm{cm})$ & \multicolumn{2}{|c|}{40} & \multicolumn{2}{|r|}{40} \\
\hline & Textura del suelo & \multicolumn{2}{|c|}{ Franco arenoso } & \multicolumn{2}{|c|}{ Franco limoso } \\
\hline \multicolumn{2}{|c|}{ Intervalo de humedad inicial } & \multicolumn{2}{|c|}{$80 \%$} & \multicolumn{2}{|r|}{$80 \%$} \\
\hline \multicolumn{6}{|c|}{ Datos del sistema de riego y manejo } \\
\hline & & \multicolumn{2}{|c|}{ Cebolla } & \multicolumn{2}{|c|}{ Maíz } \\
\hline \multicolumn{2}{|r|}{ Sistema de riego } & \multicolumn{2}{|c|}{ Aspersión-Cobertura } & \multicolumn{2}{|c|}{ Aspersión-Cobertura } \\
\hline \multicolumn{2}{|c|}{ Pérdidas por evaporación y arrastre } & \multicolumn{2}{|c|}{$9 \%$} & \multicolumn{2}{|c|}{$9 \%$} \\
\hline \multicolumn{2}{|c|}{ Coeficiente de uniformidad } & \multicolumn{2}{|c|}{$88 \%$} & & $88 \%$ \\
\hline Máximo y $n$ & í́nimo volumen en un riego (mm) & 22,8 y & 5,7 & 25, & 65 y 5,7 \\
\hline Máximo & $\begin{array}{l}\text { / mínimo intervalo entre riegos } \\
\text { (días) }\end{array}$ & $3 y$ & & & 5 у 2 \\
\hline & & tros datos & & & \\
\hline & s. Fyanotransnir & ia $(\mathrm{mm})$ y $\mathrm{p}$ & & & \\
\hline
\end{tabular}

Como se ha indicado, a partir de los datos obtenidos de la programación de riegos, se ha determinado el tiempo de apertura de cada hidrante. Para establecer la hora de apertura, se han planteado tres escenarios: a) El Escenario 1, que considera que todas las tomas están abiertas en el mismo momento, tomando como hora de inicio la misma en todas ellas; b) El Escenario 2, donde la mitad de las tomas se ponen en marcha a una misma hora (igual hora de inicio) mientras que para el resto, la hora de apertura se establece de modo aleatorio; c) Escenario 3, donde la hora del inicio del riego en todas las parcelas se establece de forma aleatoria. Estos escenarios se han planteado teniendo en cuenta aspectos de manejo característicos de la zona, tales como que, normalmente, justo en el comienzo del tramo tarifario más económico (P6) gran parte de las parcelas comienzan a 
regar. El horario de riego se ha limitado al período de menor coste económico (P6), por lo que la red sólo estará en funcionamiento entre las 0:00 y las 8:00. Para analizar el comportamiento hidráulico de la red en función del tiempo, se han considerado períodos de cálculo cada 15 minutos y el coste energético de las tarifas contratadas por la sociedad (Tabla 3).

Tabla 3. Tarifas y precios de coste de energía para el mes de julio.

\begin{tabular}{|c|c|c|c|c|}
\hline \multirow{3}{*}{ Mes } & \multicolumn{2}{|c|}{ Horario } & \multirow{2}{*}{ Tarifa } & \multirow{2}{*}{ Coste (€/kW h) } \\
\cline { 2 - 3 } & Inicial & Final & & \\
\hline \multirow{4}{*}{ Julio } & $0: 00$ & $8: 00$ & P6 & 0,053428 \\
\cline { 2 - 5 } & $8: 00$ & $10: 00$ & P4 & 0,080755 \\
\cline { 2 - 5 } & $10: 00$ & $14: 00$ & P3 & 0,087611 \\
\cline { 2 - 5 } & $14: 00$ & $18: 00$ & P4 & 0,080755 \\
\cline { 2 - 5 } & $18: 00$ & $22: 00$ & P3 & 0,087611 \\
\cline { 2 - 5 } & $22: 00$ & $0: 00$ & P4 & 0,080755 \\
\hline
\end{tabular}

La evaluación del comportamiento de la red para los distintos escenarios se apoyó en el modelo calibrado de la misma, realizado en trabajos previos (Moreno et al, 2007) y el motor de cálculo EPANET® (Rossman, 2001), en combinación con otras herramientas desarrolladas en MATLAB $®$. Para cada escenario se caracterizó el punto de funcionamiento del grupo de bombeo (caudal y presión), el rendimiento medio de la estación de bombeo, así como el consumo energético y su coste. Para determinar el rendimiento medio del grupo de bombeo, se utilizó el Modelo de Análisis de Eficiencia Energética en Estaciones de Bombeo (MAEEB) (Moreno et al, 2007).

\section{3) Resultados y discusión}

Con respecto a los caudales requeridos en cabecera, como es obvio, el volumen acumulado fue el mismo para los distintos escenarios. Por otra parte, la distribución de los caudales es distinta (Fig. 2), ya que tanto la hora de apertura, como número de tomas abiertas simultáneamente, cambió.

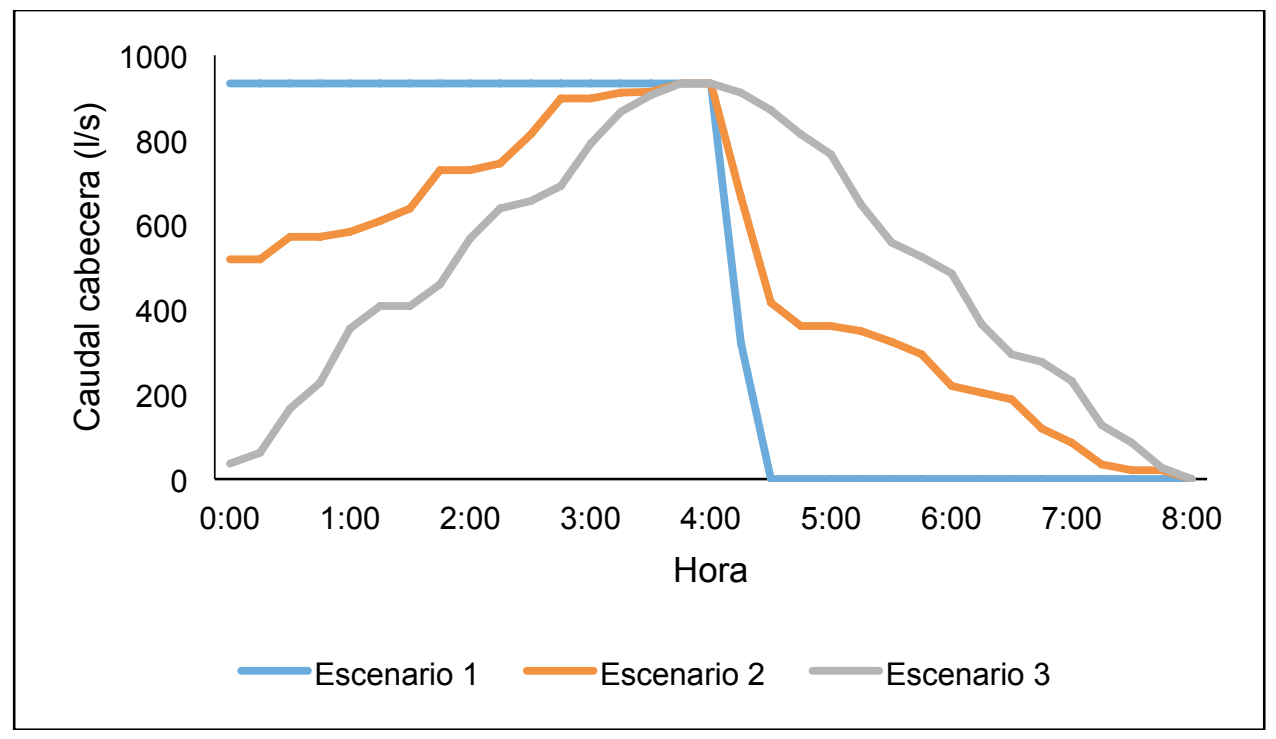

Figura 2. Caudal en cabecera para los distintos escenarios (período P6, desde las 0:00 hasta las 8:00). 
En el Escenario 1, la distribución de caudales se concentra en las cuatro primeras horas de la tarifa P6 (de las 0:00 hasta las 4:00), ya que todas las tomas están abiertas al mismo tiempo, por lo que el caudal en cabecera toma un valor constante próximo a los 900 I/s.

Para el Escenario 2, la mayor demanda se produce en la primera parte del período (entre 00:00 a 4:00 horas), con un máximo cerca de las 4:00 h (de aproximadamente 900 I/s). Esto es debido a que la mitad de las tomas están abiertas durante las primeras cuatro horas, sumando los caudales derivados de la apertura aleatoria de tomas. Por el contrario, para el Escenario 3, en el que el horario de apertura de las tomas es aleatorio, la distribución de los caudales se asemeja a una distribución normal.

La altura de presión horaria demandada en cabecera se recoge en la Figura 3. La distribución horaria de las presiones es similar a la establecida para los caudales. Así, el Escenario 1 mantienen las presiones constantes con su máximo valor (aproximadamente 60 m.c.a) durante las cuatro primeras horas del período, anulándose para el resto, mientras que en los escenarios 2 y 3 presenta variaciones de entre 40-60 m.c.a, con el valor máximo en la hora central del período.

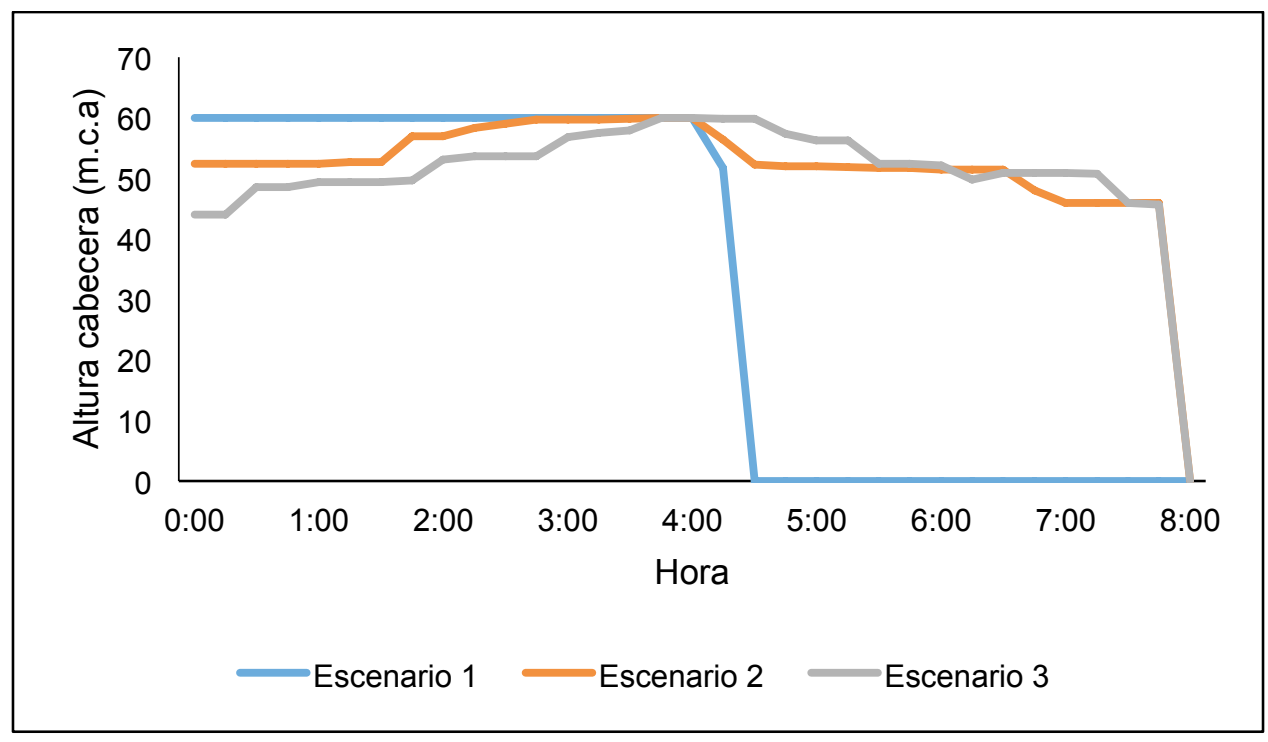

Figura 3. Presión en cabecera para los distintos escenarios.

Durante el tiempo de funcionamiento del grupo de bombeo, el mayor valor de presión media de trabajo se da en el Escenario 1, con 60 m.c.a., con valores parecidos en los Escenarios 2 y 3 , de 54 m.c.a. y de 53 m.c.a., respectivamente (Tabla 4).

Tabla 4. Presión media en cabecera (m.c.a.) para los escenarios analizados

\begin{tabular}{|c|c|c|c|c|}
\hline Escenario & Media & Mínimo & Máximo & Desviación estándar \\
\hline 1 & 59.45 & 51.76 & 59.91 & 1.92 \\
\hline 2 & 53.46 & 45.87 & 59.90 & 4.49 \\
\hline 3 & 52.50 & 43.96 & 59.91 & 4.66 \\
\hline
\end{tabular}

La eficiencia energética de la estación de bombeo, es similar en los tres casos analizados, con valores próximos al $60 \%$, siendo ligeramente superior para el primer escenario $(62,16 \%)$. En este caso, el valor es constante, mientras que para los otros oscila 
entre $35 \%$ y $71 \%$ para el Escenario 2 (valor medio del $58,41 \%$ ), y entre el $40 \%$ y $71 \%$ en el Escenario 3 (valor medio del 58,66\%).

La evolución de la energía consumida por la estación de bombeo (Fig. 4) mostró una evolución similar a la obtenida con la distribución de caudales, donde el Escenario 1 presenta un consumo constante $(211 \mathrm{KW} \mathrm{h})$. Considerando el consumo total de energía, el Escenario 1 presenta un mayor consumo, siendo del $3 \%$ y $4 \%$ superior en comparación con el Escenario 2 y 3, respectivamente.

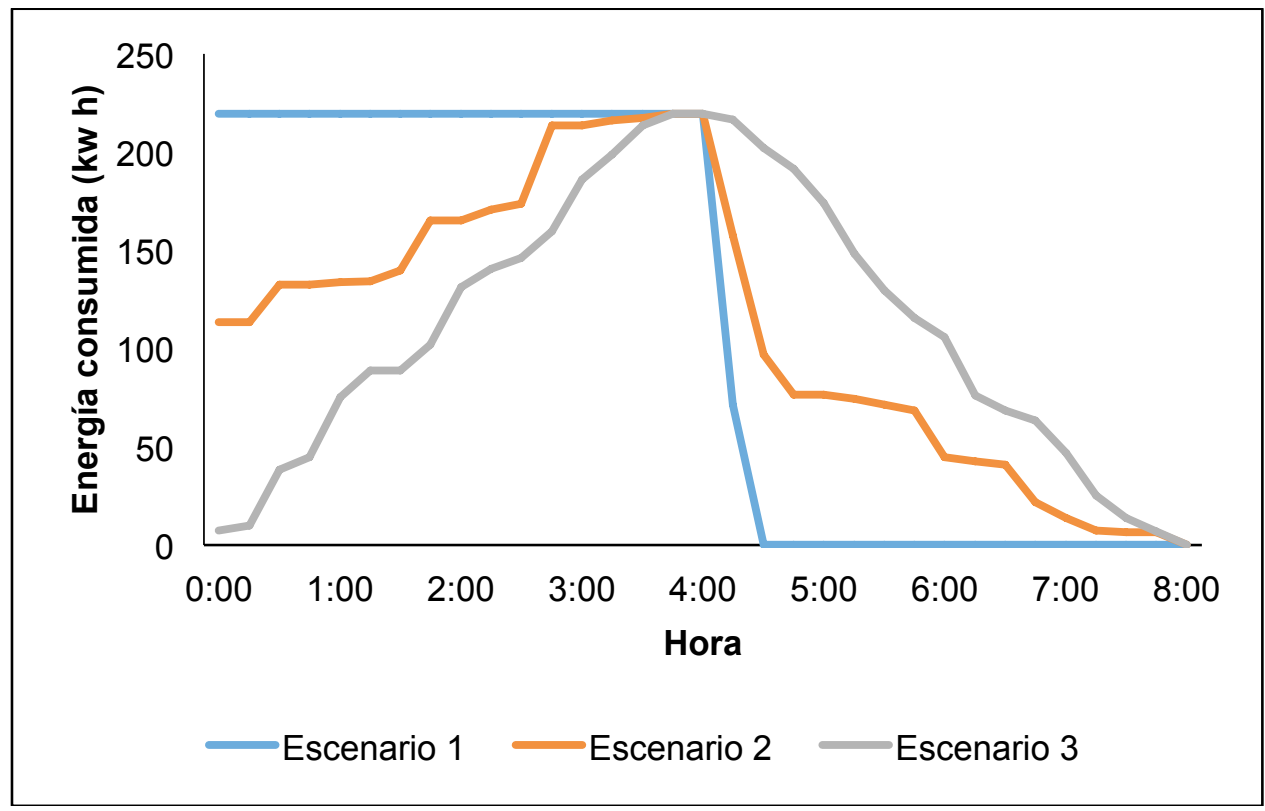

Figura 4. Consumo energético de la estación de bombeo, para los distintos escenarios.

La Figura 5, muestra los costes acumulados por hora para los distintos escenarios. Considerando el coste acumulado para el día de estudio, el Escenario 3 es el que mayor coste acumulado presenta $(203 €)$. Con respecto a éste, en los Escenarios 2 y 3 se estiman ahorros del $3 \%(196 €)$ y el $4 \%(195 €)$.

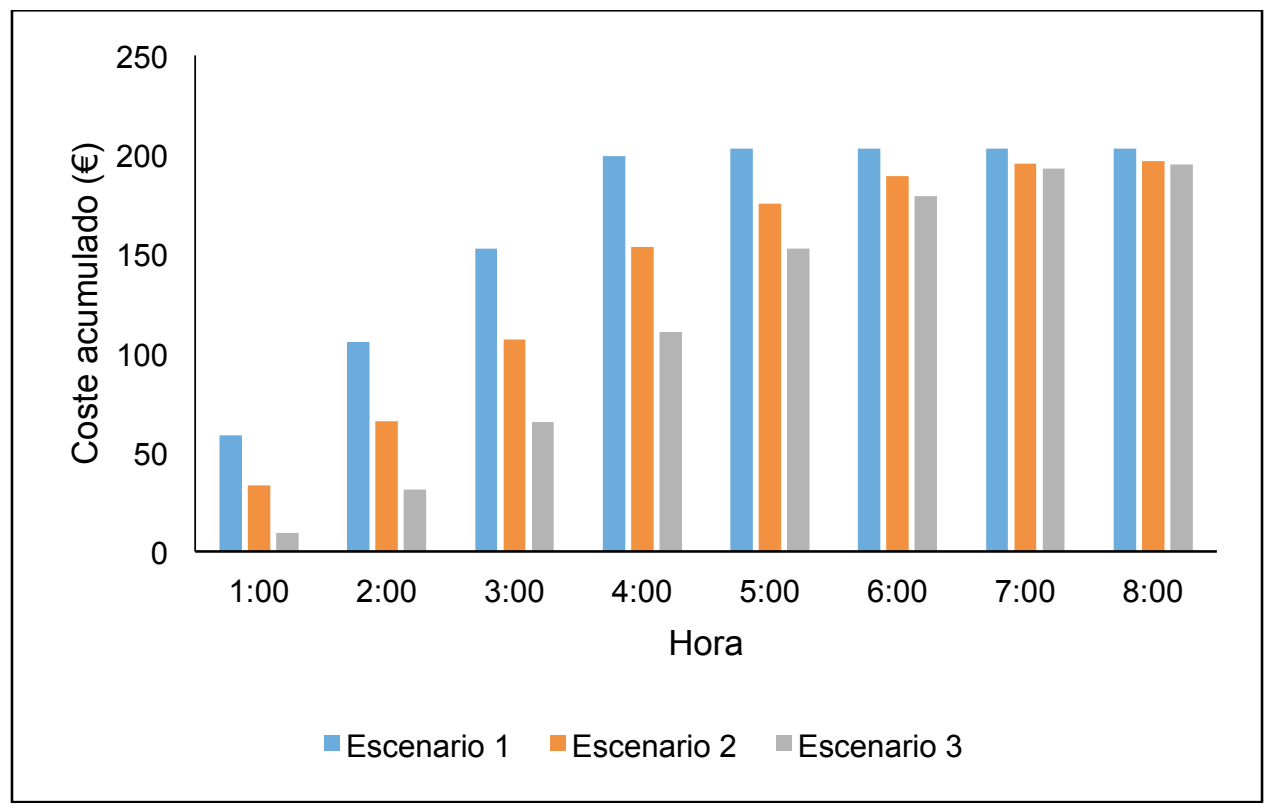

Figura 5. Coste acumulado por hora para los distintos escenarios. 


\section{4) Conclusiones y Recomendaciones}

La optimización del manejo de una red colectiva de riego exige controlar y conocer tanto el comportamiento de la red, como la programación de riego en parcela. Así, es posible prever el funcionamiento de la red a partir de los requerimientos hídricos derivados de la programación de los riegos en parcela. La variación de la secuencia de apertura y cierre de los hidrantes no modifica el agua suministrada a los cultivos, pero permite establecer un escenario que minimice el coste energético en la estación de bombeo. Este aspecto está relacionado con el hecho de que distintos manejos de la red conllevan a distintos comportamientos de la misma desde el punto de vista energético.

Entre los escenarios planteados en este trabajo, la concentración del horario de apertura de los hidrantes (Escenario 1) conlleva un incremento del consumo energético de la red, disminuyendo cuando la apertura de las tomas se produce de modo aleatorio (Escenario 3).

La integración de la programación de riegos en el manejo conjunto de la red por un gestor permitirá disminuir los costes energéticos sin reducir los aportes de agua recibidos por los cultivos.

\section{5) Bibliografía}

Allen, R.G. Pereira, L.S. Raes, D. Smith,M. 1998. Crop evapotranspiration: guidelines for computing crop water requirements. FAO. Irrigation and Drainage Paper, 56, 297.

Carrillo-Cobo, M, T,, Rodríguez, J,A,, Montesinos, P,, López, R,, Camacho, E,, 2011, Low energy consumption seasonal calendar for sectoring operation in pressurized irrigation networks, Irrig, Sci, 29, 157-169.

Domínguez, A, Juan, J,A, Tarjuelo, J,M, Martínez, R,S, Martínez-Romero, A, (2012a), Determination of optimal regulated deficit irrigation strategies for maize in a semi-arid environment, Agricultural Water Management, 110, 67-77.

Domínguez, A, Jiménez, M, Tarjuelo, J,M, Juana, J,A, Martínez-Romeroa, A, Leite, K,N, (2012b), Simulation of onion crop behavior under optimized regulated deficit irrigation using MOPECO model in a semi-arid environment, Agricultural Water Management, $113,64-75$.

Domínguez, A. Martínez-Romero, A. Leite, K.N. Tarjuelo, J.M. Juana, J.A. López-Urreac, R. 2013. Combination of typical meteorological year with regulated deficit irrigation to improve the profitability of garlic growing in central spain. Agricultural Water Management, 130, 154-167.

Fernández-García I, Moreno MA, Rodríguez-Díaz JA (2014a) Optimum pumping station management for irrigation networks sectoring: Case of Bembezar MI (Spain), Agric Water Manage 144:150-158.

Gonzalez Perea, R,, Camacho Poyato, E,, Montesinos, P,, Rodríguez-Díaz, J,A, 2014, Critical points: interactions between on-farm irrigation systems and water distribution network, Irrig, Sci, 32: 255-265.

MAGRAMA, 2015, Red del Servicio de Información Agroclimática para el Regadío (SIAR), Ministerio de Agricultura, Alimentación y Medio Ambiente (http://www,magrama,gob,es/).

Moreno, M, A,, Pelayo, J,, Córcoles, J, I,, Planells, P,, Carrión, P, A,, Tarjuelo, J, M,, 2007, Measurement and improvement of the energy efficiency at pumping stations. Biosys, Engin, 98:479-486. 
Rossman, L, A, (2001), EPANET 2, Users Manual, Water supply and Water Resources Division National Risk Management Research Laboratory, Cincinati, USA: U,S, Enviromental Protection Agency. 